\title{
Digital Image Watermarking using Fibonacci Transform in YIQ Color Space
}

\author{
Manoranjan Kr Sinha \\ Dept.of E.C.E. \\ NIRT-Bhopal, India
}

\author{
Rajesh Kumar Rai, PhD \\ Dept.of E.C.E. \\ NIRT-Bhopal, India
}

\author{
G. Kumar \\ Dept.of E.C.E. \\ NIRT-Bhopal, India
}

\begin{abstract}
Digital watermarking is a technique for inserting ownership information to the digital data to prove the authenticity. A digital watermarking is perceptually invisible to prevent obstruction of the original image. It is also used for tamper proofing, broadcast monitoring, covert communication etc. In this paper, a wavelet-based watermarking approach for hiding watermark image in color host images is proposed. The host image is converted into YIQ color space and then Y channel decomposed into wavelet coefficients. This proposed methodology overcomes the drawback of the existing approaches and enhances the security by applying Arnold transform and Fibonacci transform for embedding the resultant pattern in the host image. The watermark embedding process does not degrade the visual quality of the image.
\end{abstract}

\section{General Terms}

Image Processing, Watermarking, YIQ Color Space, MSE, PSNR.

\section{Keywords}

3-level DWT, Arnold transform, Fibonacci Transform.

\section{INTRODUCTION}

Over the past few years, there has been tremendous growth in computer networks, communication technology and specifically, the World Wide Web. These technologies uses multimedia data like image, video for transmission of digital data, these can be easily duplicated and distributed without the owner's consent. This result in unauthorized copying and distribution of digital data that infringe the intellectual property right of the owners. Using some encryption technique to protect the data is a possible solution to the problem. But encryption does not provide over all protection. Once the encrypted data are decrypted they are freely distributed or manipulated. Digital watermarking has been emerged as an effective solution to the problem. It is a technique for inserting ownership information to the digital data to prove the authenticity. It is also used for tamper proofing, broadcast monitoring, covert communication etc. A digital watermark is a type of code or image incorporated in a noise-tolerant signal such as audio or image data which is used to identify ownership of the copyright of that signal or content of the document for authentication. Digital watermarks are visible and invisible but invisible watermark is preferred since it does not cause perceptual degradation of host signal. Another requirement of watermark is that it should be robust against attacks, i.e., it should survive signal processing operations and counterfeit attempts. A high watermarking capacity is another major requirement. In other words it should carry as many bits of information as possible. Watermark is embedded onto the signal in spatial domain or frequency domain. In frequency domain signal is transformed by using transforms such as discrete cosine transform (DCT), discrete fourier transform (DFT), discrete wavelet transform (DWT) and the watermark is embedded onto the coefficients obtained in frequency domain.

Each watermarking application has its own specific requirements. Some of the general requirements are as follows: [1]

Perceptual transparency: the watermarking algorithm must embed the watermark such that this does not affect the quality of the host data. A watermark-embedding procedure is truly imperceptible if humans cannot distinguish the original data from the data with the inserted watermark [2].

The smallest modification in the host data becomes apparent when the original data is compared directly with the watermarked data. Since users of watermarked data normally do not have access to the original data, they cannot perform this comparison. Therefore, the modifications in the watermarked data go unnoticed as long as the data are not compared with the original data [3].

Watermarking load: The amount of information that is stored in a watermark depends on the application. The information should be embedded such that the pixel value of image does not leave the base value of the pixel.

Robustness: There should be no way in which the watermark is removed or altered without sufficient degradation of the perceptual quality of the host data. If a watermark is used for another application, it is desirable that the watermark always remains in the host data, even if the quality of the host data is degraded, intentionally or unintentionally [4].

Security: A watermarking technique is secure if knowing the exact algorithms for embedding and extracting the watermark does not help an unauthorized party to detect the presence of the watermark [1].

Blind detection scheme. Non-blind detection needs the original host signal, which is very inconvenient to use the original data, because of the huge video data. Blind detection does not need the original host image.

High real-time: Three-dimensional video signal has more the amount of data than the image does. So calculation quality is larger and embedding/detection needs more times. The procession of embedding, using video compression standard for these specific structures such as motion vector coding.

Random detection: The watermark is detected in any position of the video rather than the position according to the video playback order to detect the watermark.

Figure1 shows the watermark transmission. The watermark W is generated as a pseudo-random sequence to ensure statistical 
invisibility. Signal information, such as DCT/DWT coefficients are extracted from the original image I and embedded into the information to form the watermarked image $\mathrm{J}$. threshold value. If the value of $\mathrm{M}$ is larger than the threshold value, then the watermark is detected.

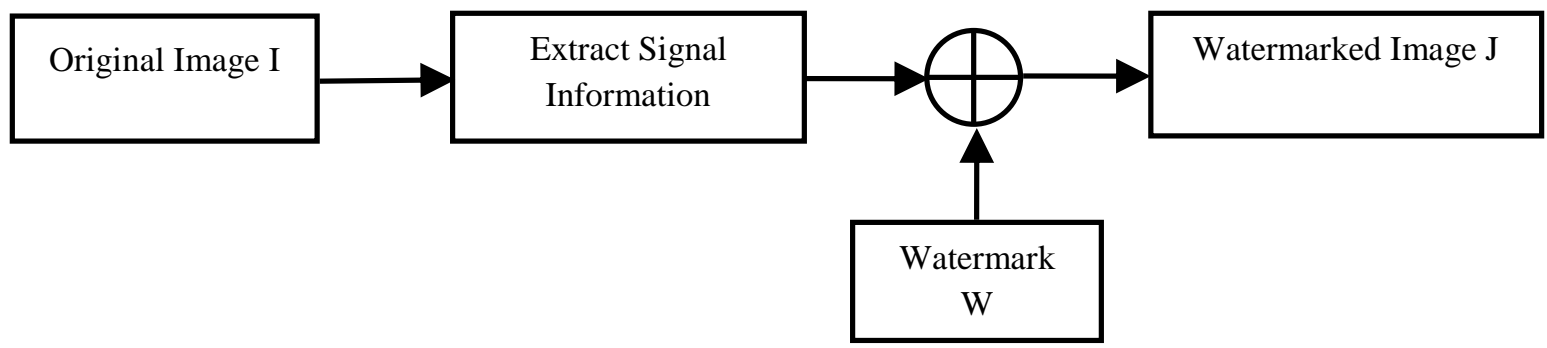

Figure 1. Watermark Transmission

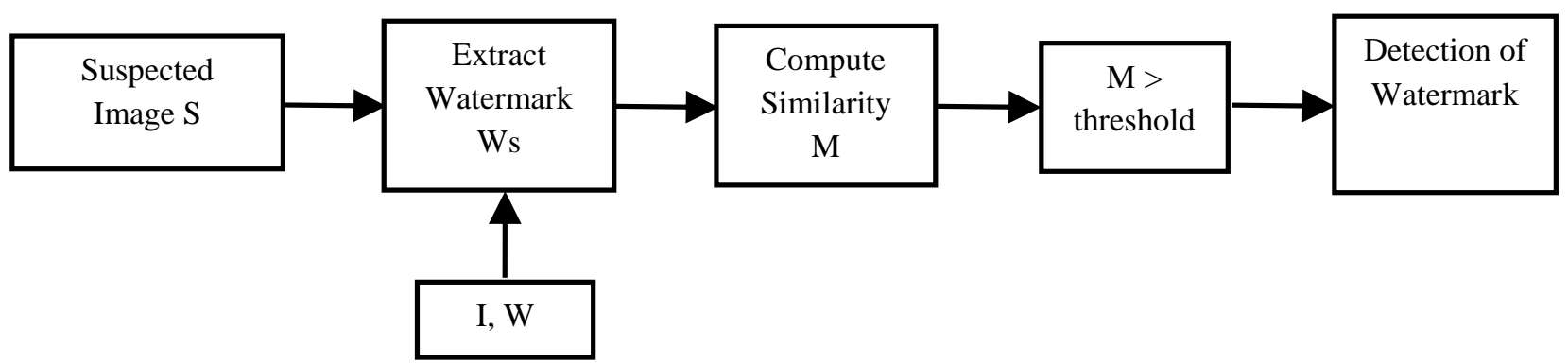

Figure 2. Watermark Detection

\section{LITERATURE REVIEW}

Recent researchers on secure digital watermarking techniques have revealed the fact that the content of the images is used to improve the invisibility and robustness of a watermarking scheme. In this approach, watermark is created from the content of the host image and discrete wavelet transform (DWT) is used for embedding watermarks, since it is an excellent time-frequency analysis method which is adapted well for extracting the information content of the image [5].

Wang et al. [6] adopt a key dependent wavelet transform. To take the advantage of localization and multi-resolution property of the wavelet transform, Wang and Lin [2] proposed wavelet tree based watermarking algorithm. In this approach, the host image is transformed into wavelet coefficients using a discrete-time wavelet transform (DTWT). The watermark is embedded in the wavelet coefficients which are grouped into super trees. Each watermark bit is embedded using two super trees. Depending on the value of the watermark bit, one of the super trees is quantized with respect to a quantization index in such a way that the two super trees exhibit a large enough statistical difference, which is extracted for obtaining decision. As each watermark bit is embedded in various frequency bands and the information of the watermark bit is spread throughout large spatial regions, therefore the watermarking technique is robust to attacks in both frequency and time domains. This technique is useful for removal of high-pass details in JPEG compression and robust to time domain attacks such as pixel shifting and rotation. Figure 2 shows the watermark detection. In watermark detection a suspected image $\mathrm{S}$ is taken as input for obtaining its signal information. A suspected watermark $\mathrm{W}_{\mathrm{S}}$ is extracted based on knowledge of the original image $\mathrm{I}$ and the watermark W. A similarity measure $\mathrm{M}$ is calculated based on the values of $\mathrm{W}_{\mathrm{S}}$ and $\mathrm{W}$, which is then compared with a
With copyright protection, the proposed watermarking scheme can also be applied to data hiding or image authentication.

Tao et al. [7] proposes a discrete-wavelet transform based multiple watermarking algorithms. In this approach, two important tools encryption and watermarking is used to prevent unauthorized consumption and duplication. The watermark is embedded into LL and $\mathrm{HH}$ sub-bands to improve the robustness. This approach is useful in such a way that embedding the watermark in lower frequencies is robust to a group of attacks such as JPEG compression, blurring, adding Gaussian noise, rescaling, rotation, cropping, pixilation, sharpening and embedding the watermark in higher frequencies is robust to another set of attacks such as histogram equalization, intensity adjustment, gamma correction.

Luo et al. [8] introduced an integer wavelet based watermarking techniques to protect the copyright technique to enhance the security. This technique is useful for digital watermarking in DEM (digital elevation mode) data, which effectively protects the copyright of DEM data and avoids the unauthorized user. As lifting based scheme is added to construct the compactly supported wavelets whose coefficients are composed of a tree variable therefore, it uses only integral addition and shift which is fast and easily realized via hardware. As wavelet coefficient set is embed watermark information, therefore the bit is inserted in the high activity texture regions with the maximum strength of Just Noticeable Distortion (JND) tolerance of Human Visual System (HVS) that makes the digital watermark robust.

Yuan et al. [9] proposed an integer wavelet based multiple logo watermarking scheme. The watermark is permutated using Arnold transform and is embedded by modifying the 
coefficients of the HH and LL sub-bands. In this approach, an integer wavelet based multiple logo-watermarking schemes for copyright protection of digital image is presented. A visual meaningful binary logo is used as watermark. The process of watermark embedding is carried out by transforming the host image in the integer wavelet domain. To construct a blind watermarking scheme, wavelet coefficients of $\mathrm{HH}$ and $\mathrm{LL}$ bands are modified depending on the watermark bits. To add the security, permutation is used to preprocess the watermark. Lin et al. [10] put forward a DWT based blind watermarking scheme by scrambling the watermark using chaos sequence. Watermarking in DWT domain has drawn extensive attention for its good time-frequency features and its accurate matching of the human visual system (HVS).

Chen et al. [11] proposed two DWT-based audio watermarking algorithms that one of them is based on optimization scheme using group-amplitude quantization and the other embeds information by energy-proportion scheme. Therefore, normalized energy is used instead of probability which rewrites the entropy in information theory as energyproportion function.

Preda et al. [12] proposed three DWT-based video watermarking approaches in which the watermarks used are binary images. Although, in one of them a spread-spectrum technique is used to spread the power spectrum of the watermark data, in the two others, watermarking methods are based on a combination of spread spectrum and quantization.

Deng and Jiang [13] proposed a DWT-based image watermarking algorithm in which the code-division multiple access (CDMA) encoded binary watermark, adaptively is embedded into the third level detail sub-band of DWT domain.

It is clear from the literature survey that many of the algorithms proposed met the imperceptibility requirement quite easily but robustness to different image processing are mainly applied to content authentication attacks is the key challenge and the algorithms in literature addressed only a subset of attacks.

\section{RESEARCH FINDINGS}

The existing approach applies correlation based image watermarking algorithms with respect to changes in watermarking anticipating properties such as imperceptibility and robustness against different attacks.

For security, the binary watermark image scrambled which is reshaped to a sequence and then a random binary sequence is adopted to encrypt the watermark. This process uses a pseudo-random number generator to determine the pixel to be used on a given key.

The RGB channels of the host image are converted to the intended channels and then the first channel is pre-filtered to enhance embedding process.

Low frequency sub-band of wavelet decomposition of its first channel is quantized and divided to different sub-blocks with the certain sub-block size to embed the encrypted watermark.

\subsection{Discrete Wavelet Transform}

The wavelet transform describes a multi-resolution decomposition process in terms of expansion of an image into a set of wavelet basis functions. Discrete wavelet transformation has its own space frequency localization property [14]. The input image is divided into four nonoverlapping multi-resolution sub-bands by the filters, namely LL1 (approximation coefficients), LH1 (vertical details), HL1 (horizontal details) and HH1 (diagonal details). The sub-band (LL1) is processed further to obtain the next coarser scale of wavelet coefficients, until some final scale ' $\mathrm{N}$ ' is reached. When ' $\mathrm{N}$ ' is reached, $3 \mathrm{~N}+1$ sub-band are obtained consisting of the multi-resolution sub-bands, which are LLX and LHX, HLX and HHX, where ' $X$ ' ranges from 1 until ' $N$ '. Generally, most of the image energy is stored in the LLX sub-bands.

As the image energy is concentrated at the lower frequency sub-band LLX and therefore the embedding process in such sub band degrades the performance of the image. However embedding in low frequency increases the robustness, whereas high frequency HHX includes the edges and textures of the image and human eyes are not sensitive to the changes therefore allows performing embedding without human perception.

\subsubsection{Characteristics of DWT}

(i). Wavelet Transform is computationally efficient and implemented by using simple filter convolution.

(ii). Image is represented at more than one resolution level with multi-resolution analysis. Wavelets allow image to be described in terms of coarse overall shape and details ranging from broad to narrow.

(iii). Magnitude of DWT coefficients is larger in the lowest bands (LL) at each level of decomposition and is smaller for other bands (HH, LH, HL).

(iv). The larger the magnitude of wavelet coefficient, the more significant it is.

(v). Watermark detection at lower resolutions is computationally effective because at every successive resolution level, less number of frequency bands are involved.

(vi). High resolution sub bands help to easily locate edge and textures patterns in an image.

\subsubsection{Advantages of DWT}

Wavelet transform is considered for image watermarking for such reasons:

Wavelet transform can accurately model HVS than other transforms like Discrete Fourier Transform (DFT) [9] or Discrete Cosine Transform (DCT) [14, 15]. This allows higher energy watermarks in regions where HVS is less sensitive. Embedding watermark in these regions allow to increase robustness of watermark, with no much degradation of image quality.

Wavelet coded image is a multi-resolution description of image. Hence an image can be shown at different levels of resolution and can be sequentially processed from low resolution to high resolution. The advantage of such approach is that the features of an image that might go undetected at one resolution are easy to spot at another.

Visual artifacts introduced by wavelet coded images are less evident compared to DCT because wavelet transform doesn't decompose image into blocks for processing. At high compression ratios, blocking artifacts are noticeable in DCT as against wavelet transformed images.

DFT and DCT are full frame transform. Hence, any change in the transform coefficients affects entire image except if DCT is implemented using a block based approach. However DWT 
has spatial frequency locality. It affects the image locally, if watermark is embedded.

To perform third level decomposition, the DWT is applied to LL2 band which decompose this band into the four sub-bands - LL3, LH3, HL3, HH3. This results in 10 sub-bands per component. LH1, HL1, and HH1 contain the highest frequency bands present in the image tile, while LL3 contains the lowest frequency band.

The three-level DWT decomposition is shown in Figure3.
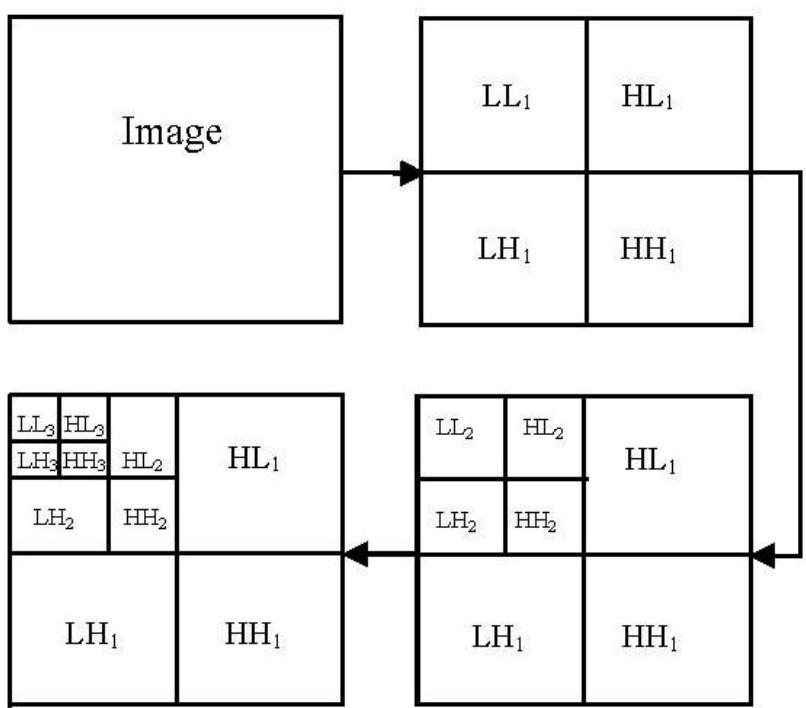

Figure 3. DWT Watermark with three levels

\subsection{YIQ Color Space}

The National Television Systems Committee (NTSC) defines a color space known as YIQ which is used in televisions in the United States. One of the main advantages of this format is that grayscale information is separated from color data, so the same signal is used for both color black and white sets.

In the YIQ color space Y component represent the intensity, the I and Q component represents the color information. By separating the intensity from the color information makes the YIQ color space very attractive to TV broadcasting, because it helps maintain compatibility with monochrome TV standards. The first component, luminance, represents grayscale information, while the last two components make up chrominance (color information).

The YIQ model has advantage of the fact that the human eye is more sensitive to changes in luminance than changes to hue or saturation.

The Y, I, Q components are assumed to be in the $[0,1]$ or $[0$, 255] range.

The RGB to YIQ conversion is defined as:

$$
\left[\begin{array}{l}
\mathrm{Y} \\
\mathrm{I} \\
\mathrm{Q}
\end{array}\right]=\left[\begin{array}{ccc}
0.299 & 0.587 & 0.114 \\
0.595716 & -0.274453 & -0.321263 \\
0.211456 & -0.522591 & 0.311135
\end{array}\right]\left[\begin{array}{l}
\mathrm{R} \\
\mathrm{G} \\
\mathrm{B}
\end{array}\right]
$$

The YIQ to RGB conversion is defined as:

$$
\left[\begin{array}{l}
\mathrm{R} \\
\mathrm{G} \\
\mathrm{B}
\end{array}\right]=\left[\begin{array}{ccc}
1 & 0.9563 & 0.6210 \\
1 & -0.2721 & -0.6474 \\
1 & -1.1070 & 1.7046
\end{array}\right]\left[\begin{array}{l}
\mathrm{Y} \\
\mathrm{I} \\
\mathrm{Q}
\end{array}\right]
$$

To obtain the RGB values from a set of YIQ values, the inverse matrix operation is performed.
The function rgb2ntsc converts colormaps or RGB images to the NTSC color space and ntsc2rgb performs the reverse operation.

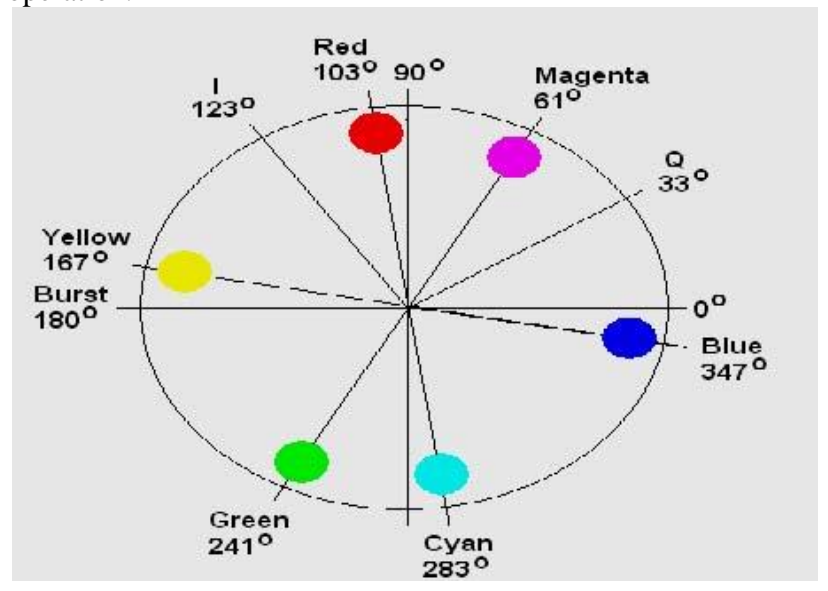

Figure 4. YIQ Representation

For example, Commands convert an RGB image to NTSC format are.

RGB = imread('man.png');

$\mathrm{YIQ}=\operatorname{rgb} 2 \mathrm{ntsc}(\mathrm{RGB})$;

Because luminance is one of the components of the NTSC format, the RGB to NTSC conversion is also useful for isolating the gray level information in an image. The toolbox functions rgb2gray and ind2gray use the rgb2ntsc function to extract the grayscale information from a color image.

\subsection{Arnold's Transformation}

Arnold Transform is commonly known as cat face transform and is mainly suitable for digital images of size $\mathrm{N} \mathrm{x} \mathrm{N}$. It is defined as $\left(\begin{array}{l}x^{\prime} \\ y^{\prime}\end{array}\right)=\left(\begin{array}{l}11 \\ 12\end{array}\right)\left(\begin{array}{l}x \\ y\end{array}\right) \bmod \mathrm{N},(\mathrm{x}, \mathrm{y})$ and $\left(\mathrm{x}^{\prime}, \mathrm{y}^{\prime}\right)$ $\varepsilon\{0,1,2, \ldots \ldots \ldots \ldots \ldots . . . . . .1\}$, where $(\mathrm{x}, \mathrm{y})$ are the coordinates of original image and ( $\left.x^{\prime}, y^{\prime}\right)$ are the coordinates of image pixels of the transformed image. Transform changes the position of pixels and if done several times, scrambled image is obtained. $\mathrm{N}$ is the height or width of the square image to be processed. Arnold Transform is periodic in nature. The decryption of image depends on transformation periods. Period changes in accordance with size of image. Iteration number is used as the encryption key. When Arnold Transformation is applied, the image can do iteration, iteration number is used as a secret key for extracting the secret image.

The security of watermarking is enhanced by scrambling or encrypting before watermarking is embedded. In this paper, the extended Arnold transform is used to scramble watermarking of copyright protection. Initial Arnold transform equation is as written:

$$
\begin{aligned}
X_{n+1} & =\left(X_{n}+Y_{n}\right) \bmod 1 \\
Y_{n+1} & =\left(X_{n}+2 Y_{n}\right) \bmod 1 \\
&
\end{aligned}
$$

where, mod 1 denotes the fractional part.

The phase space of $\left(X_{n}, Y_{n}\right)$ is restricted in square $[0,1] X[0$, 1]. It transforms (1) to matrix form.

$$
\left(\begin{array}{l}
X_{n+1} \\
Y_{n+1}
\end{array}\right)=\left(\begin{array}{l}
a b \\
c d
\end{array}\right)\left(\begin{array}{l}
X_{n} \\
Y_{n}
\end{array}\right)=C\left(\begin{array}{l}
X_{n} \\
Y_{n}
\end{array}\right) \operatorname{modM}
$$

Formula (2) defines matrix C. Taking note of the determinant ICI $=1$, Arnold mapping has a fixed area. At the same time it is all mapped. The character is very important. With it each watermarking pixel in different places can get a different place to embed. Arnold mapping has two typical factors to 
generate chaotic motion. One is tensile (multiplied by the matrix $\mathrm{C}$ to make $\mathrm{x}$ and $\mathrm{y}$ larger), and another is folding (make $\mathrm{x}$ and y retract unit matrix by modulus). In fact, Arnold mapping is also a chaotic mapping. Generalize Arnold mapping as follows. Firstly, generalize phase space to $\{0, I, 2$, $\ldots, \mathrm{M}-\mathrm{I}\} \mathrm{X}\{0, \mathrm{I}, 2, \ldots, \mathrm{M}-\mathrm{I}\}$. That is, only take positive integers from ${ }^{\circ}$ to M-I. Secondly, generalize the equation as most common two-dimensional and fixed area.

Considering every pixel coordinate $(\mathrm{x}, \mathrm{y})$ of watermarking as the initial value; take three independent parameters of coefficient matrix $\mathrm{C}$ and the times of iterations $(\mathrm{k})$ as secret key; and take the result of iteration $\left(x^{\prime}, y^{\prime}\right)$ as the corresponding location where (x, y) is scrambled. Since the chaotic characteristics of mapping, the corresponding location of any two adjacent pixels scrambled of watermarking is a great deal of separation. Because it is a one by one mapping, the scrambling location got by different iteration of watermarking coordinates is different. Thus, scrambling location does not produce a conflict [16]. decomposition and remove mark. The parameter $p$ is secretly shared between the transmitter and receiver.

\section{PROPOSED WORK \\ 4.1 Problem Formulation}

The existing approach reveals that the watermarking security is satisfactory such that the watermark is scrambled by ATM and after converting to a sequence is adopted with a random binary sequence using a pseudo-random number generator to encrypt the watermark. In addition, embedding watermark in the intended channel of each color space increased the security property.

This proposed methodology overcomes the drawback of the existing approach enhances the security by applying Arnold transform and Fibonacci transform for embedding the resultant pattern in the host image. The watermark embedding process does not degrade the visual quality of the image. The designed method makes use of discrete wavelet transform which provides a frequency spread of the watermark within the host image. Moreover, the authentication process provides qualities like imperceptibility, robustness and security.

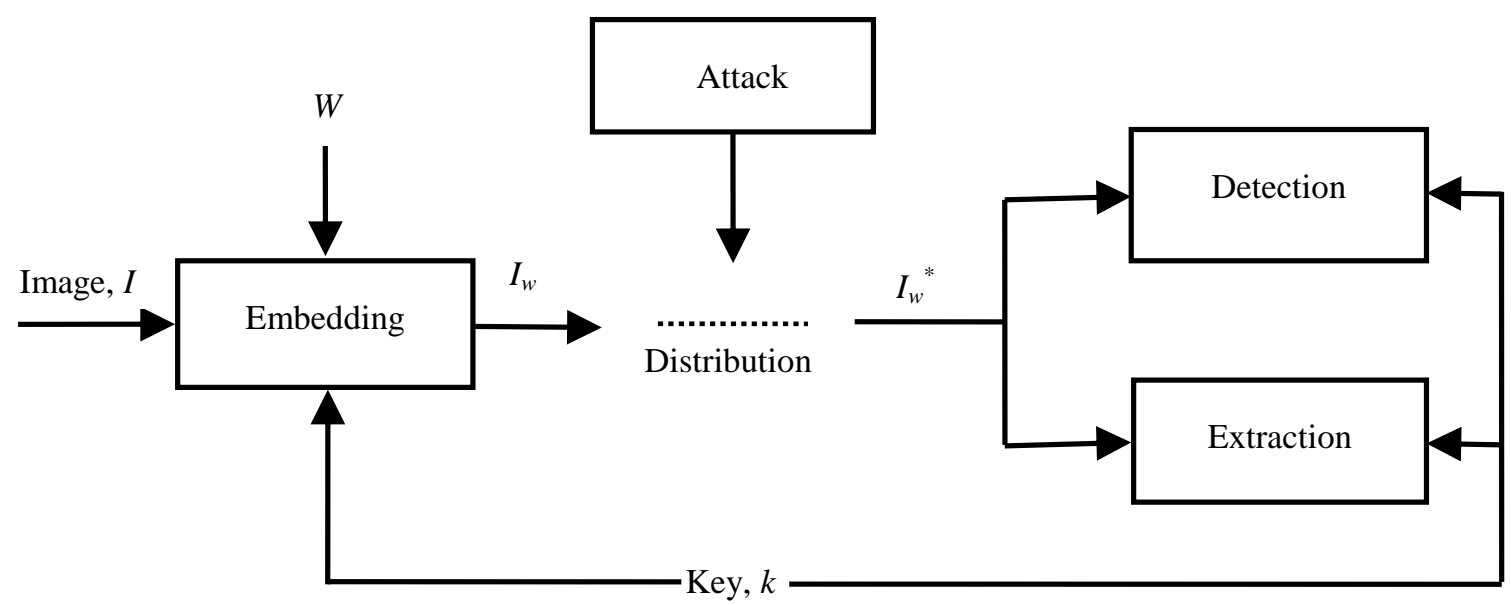

\subsection{Watermark Embedding}

Figure 5 Watermarking System

Step 1: Convert RGB channels of a host image $H$ into the intended channels.

Step 2: Pre-filtering the first channel.

Step 3: Decompose the first channel into three levels with ten DWT sub-bands, F(Y). The sub- band LL3 is taken as the target sub-band for embedding watermarks.

Step 4: Creating a sign matrix to save the signs of selected target sub-band coefficients.

\section{4 .3 Fibonacci Transformation}

To increase the security of method, a key dependent transform domain, the Fibonacci transform has been used. In Fibonacci Transform, the selection of sub band is connected to select Fibonacci. The cover images of $\mathrm{N}^{*} \mathrm{~N}, p$ is chosen sub band size.

- $\quad L L: N_{n-1} * N_{n-1}$ pixels.

- LH: $N_{n-1} * N_{n-p-1}$ pixels.

- $\quad H L: N_{n-p-1} * N_{n-1}$ pixels.

- $H H: N_{n-p-1} * N_{n-p-1}$ pixels.

For a image, the Fibonacci transform is not unique depending on the value of $p$. The value of $p$ used in the embedding process, it is difficult to obtain the exact sub band
Step 5: Quantization of the selected embedding coefficients. Step 6: Divide the target sub-band into the different subblocks.

Step 7: Determining the maximum message size of each subblocks.

Step 8: For more security of watermarks, the watermark $\mathrm{W}$ is scrambled for key times with Arnold and Fibonacci transformations and then reshaped to a sequence; after that, a random binary sequence $\mathrm{R}$ of size $\mathrm{n}$ is adopted to encrypt the watermark, where $\mathrm{n}$ is the size of the watermark image.

Step 9: Embedding the watermark using the correlation properties of additive pseudo-random noise patterns according to equation as shown:

$$
I_{W_{x, y}}(u, v)=\left\{\begin{array}{lr}
I_{x, y}+k^{*} W_{i}, & \text { if } W=0 \\
I_{x, y} * W_{i} & \text { Otherwise }
\end{array}\right.
$$

where $\mathrm{k}$ denotes a gain factor for completely controlling the imperceptibility of watermarked images and the robustness of watermarks and also Iw is the resulting watermarked image.

Step 10: Apply the sign matrix.

Step 11: Perform inverse DWT on new first channel with all changed and unchanged DWT coefficients.

Step 12: Reconvert intended channels of the changed host image into RGB channels. 


\subsection{Watermark Extraction}

Extraction algorithm is the same as embedding one and prefiltering is used before applying DWT transform to better separate watermark information from host image.

Step 1: Convert RGB channels of a watermarked image $H$ into the intended channels.

Step 2: Pre-filtering the first channel.

Step 3: Decompose the first channel into three levels with ten DWT sub-bands. The sub-band LL3 is taken as the target subband for extraction watermarks.

Step 4: Quantization of the selected embedding coefficients.

Step 5: Divide of the target sub-band into the different subblocks.

Step 6: Determining the maximum message size of each subblocks.

Step 7: Computation of threshold $\mathrm{T}$ as shown

$$
T=\frac{\text { Correlation }(H L)+\text { Correlation }(L H)}{2}
$$

Step 8: Computation of the threshold $\mathrm{T}$ and each embedded coefficient correlation in sub-blocks, separately.

Step 9: The sequence encrypted watermark is extracted as shown

$$
\left\{W_{i}=0, \text { if } C_{i}>T\right.
$$

Step 10: The encrypted image watermark is produced by reconverting the extracted sequence watermark.

Step 11: Scramble the encrypted image watermark with the same Arnold and Fibonacci algorithm with the same key times.

\section{RESULTS AND DISCUSSIONS}

To achieve the high watermark security, imperceptibility and robustness against different attacks such as JPEG compression and noise, the proposed perceptual watermarking scheme was implemented to evaluate the best color space for watermarking algorithms based on correlation techniques. In this paper, Man image of $321 \times 481$ is taken as the host images to embed a 273 x 185 binary watermark image and another host image of Lena of $512 \times 512$ as shown in Figure 6 and Figure 7.

It should be mentioned that in all of the implementations, MATLAB software is used. After watermark embedding process, the similarity of original host images and watermarked images was measured by the standard correlation coefficient is given as

$$
\text { Correlation }=\frac{\sum\left(x-x^{\prime}\right)\left(y-y^{\prime}\right)}{\sqrt{\left(x-x^{\prime}\right)^{2}} \sqrt{\left(y-y^{\prime}\right)^{2}}}
$$

Moreover, the peak signal-to-noise ratio (PSNR) was used to evaluate the quality of the watermarked images is given as

$$
P S N R=10 \log _{10} \frac{255^{2}}{M S E}(d B)
$$

where mean-square error (MSE) is defined as

$$
M S E=\frac{1}{m n} \sum_{i=1}^{m} \sum_{j=1}^{n}\left(h_{i, j}-h_{i, j}^{\prime}\right)^{2}
$$

where $\left\{h_{i, j}\right\}$ and $\left\{h_{i, j}^{\prime}\right\}$ are the grey levels of pixels in the host and watermarked images, respectively.

The larger PSNR is, the better the image quality. In general, a watermarked image is acceptable by human perception if its PSNR is $>30 \mathrm{dBs}$. In other words, the correlation is used for evaluating the robustness of watermarking technique and the PSNR is used for evaluating the transparency of watermarking technique.

The normalized correlation (NC) coefficient was used to measure the similarity between original watermarks $\mathrm{W}$ and the extracted watermarks $W^{\prime}$. It was defined as

$$
N C=\frac{\sum_{i} \sum_{j} w_{i, j} * w_{i, j}^{\prime}}{\sum_{i} \sum_{j} w_{i, j}^{2}}
$$

Figure 8 shows the watermarked images after Arnold transform; Figure 9 shows images after $3^{\text {rd }}$ level DWT transform and Figure 10 shows resultant image of Fibonacci transform in YIQ color space. As it is seen the proposed CDMA watermarking scheme yields satisfactory results in watermark imperceptibility and robustness in all color spaces. According to obtained results, it can be said that the proposed algorithm increases imperceptibility property in comparing with the similar algorithms in previous works.

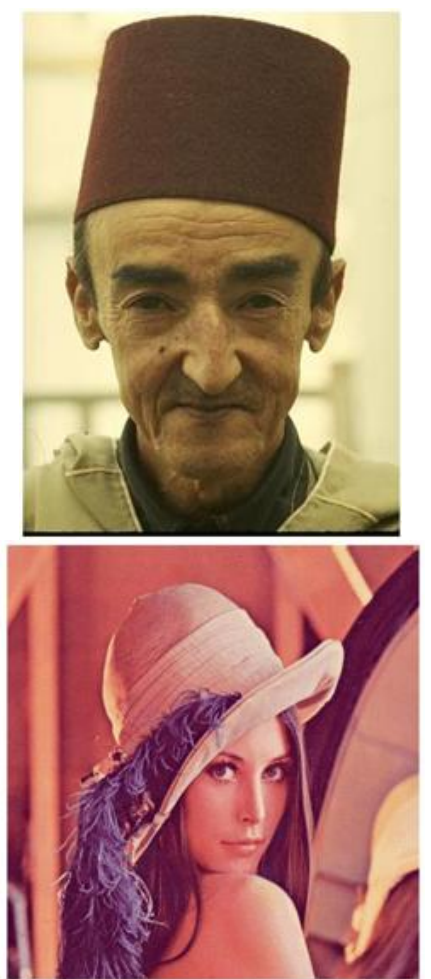

Figure 6. Original Image

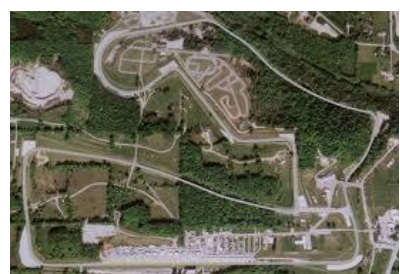

Figure 7. Watermark Image 


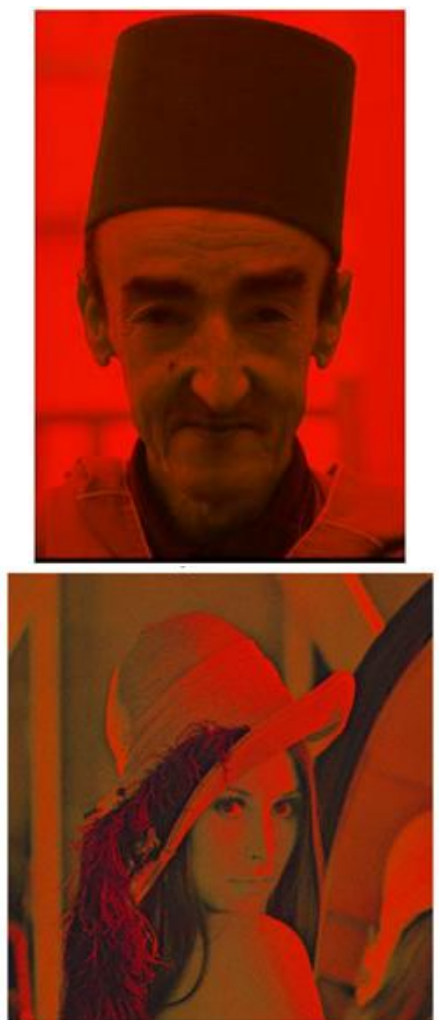

Figure 8. Image of YIQ transform

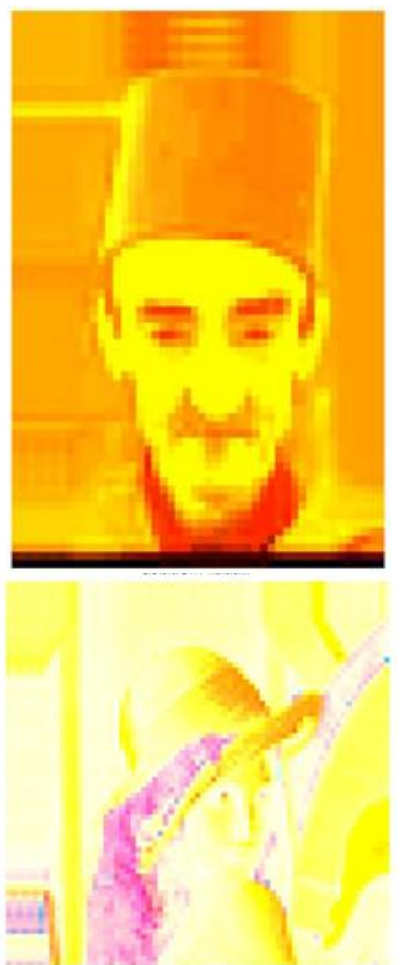

Figure 9. $3^{\text {rd }}$ level DWT transform

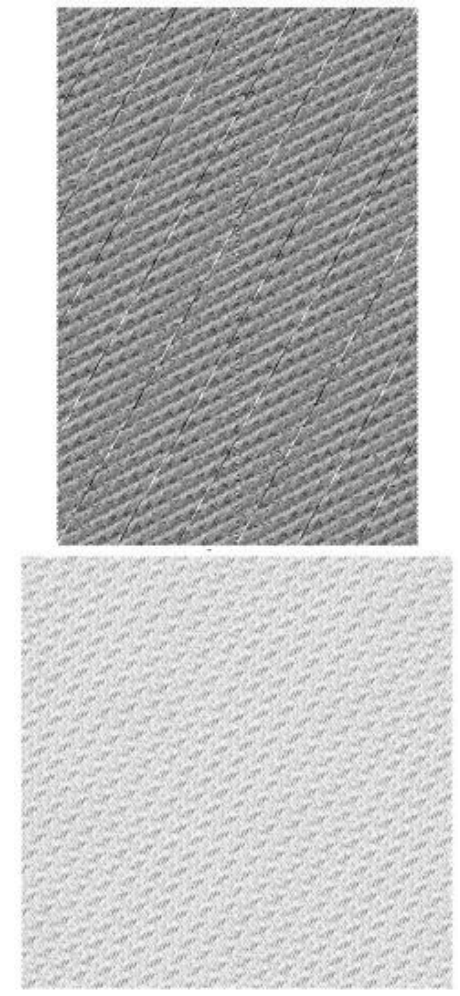

Figure 10. Resultant image after Fibonacci transform

\section{CONCLUSION}

Digital watermarking provides one of the secure ways for the protection of copyright protection with the rapid development of internet and multimedia technology. It utilizes the perceptual information of the image content to generate watermark. This proposed methodology tries to overcome the drawback of the existing approach by applying Arnold transform and Fibonacci transform for embedding the resultant pattern in the host image. The resulting image possesses a much higher readability and proves highly effective in security. In this approach, the operation of embedding and extraction of watermark is done in high frequency domains of DWT and in the absence of original image.

After watermark embedding process, the similarity of original host images and watermarked images is measured by the standard correlation coefficient. The peak signal-to-noise ratio (PSNR) is used to evaluate the quality of the watermarked images. Also the normalized correlation (NC) coefficient is used to measure the similarity between original watermarks $\mathrm{W}$ and the extracted watermarks $\mathrm{W}$ '.

Further error bit rate and affects of different types of noise attacks are calculated for further analysis of the scheme using different images in different color spaces.

\section{REFERENCES}

[1] F. Bartolini, M. Barni, V. Cappellini, and A. Piva, "Mask Building for Perceptually Hiding Frequency Embedded Watermarks," Proc. Int. Conf. on Image Processing, Oct. 1998, vol. I, pp. 450-454.

[2] Wang S.H., LinY.P., "Wavelet Tree quantization for copyright protection for Watermarking", IEEE Transactions, Image Processing, pp. 154-165, 2002.

[3] Kundur and D. Hatzinakos, "A Robust Digital Image Watermarking Method Using Wavelet-Based Fusion," 
Proc. IEEE Int. Conf. on Image Processing, Oct. 1997, vol. I, pp. 544-547.

[4] I. Cox, J. Kilian, F. Leighton, and T. Shamoon, "Secure Spread Spectrum Watermarking for Multimedia," IEEE Transactions on Image Processing, vol. 6, no. 12, pp. 1673-1687, Dec. 1997.

[5] Reddy R., et al, "Robust Digital Watermarking of Color Images under Noise Attacks", International Journal of Recent Trends in Engineering, Vol. 1, No. 1, May 2009.

[6] Wang Y., Doherty J.F., Dyck V.R.E., "A wavelet-based watermarking algorithm for ownership verification of digital images", IEEE Transactions, Image Processing, 11 pp. 77-88, 2002.

[7] Tao P., Eskicioglu A.M., "A robust multiple watermarking scheme in the discrete wavelet transform domain", Proceedings of the SPIE, Vol. 5601, pp. 133144, 2004.

[8] Luo Y., et al. "Study on digital elevation mode data watermark via integer wavelets", Journal of software, 16(6), pp. 1096-1103, 2005.

[9] Yuan Y., Huang D., Liu D., “An Integer Wavelet Based Multiple Logo- watermarking Scheme", In IEEE, Vol-2, pp. 175-179, 2006.

[10] Lin Q., Lin Z., Feng G., "DWT based on watermarking algorithm and its implementing with DSP", IEEE Xplore, pp. 131-134, 2009.
[11] Chen, S.T., Huang, H.N., Chen, C.J., Wu, G.D., 'Energyproportion based scheme for audio watermarking', IET Signal Process., 2010, 4,(5), pp. 576-587.

[12] Preda, R.O., Vizireanu, D.N., 'A robust digital watermarking scheme for video copyright protection in the wavelet domain', Measurement, 2010, 43, (10), pp. $720-1726$.

[13] Deng, N., Jiang, C.S., 'CDMA watermarking algorithm based on wavelet basis'. Proc. 9th Int. Con. Fuzzy Systems and Knowledge Discovery, May 2012, pp. $2148-2152$.

[14] Ho, C.K. and Li, C.T. Semifragile watermarking scheme for authentication of JPEG images. Proceeding of the IEEE international Conference on Information Technology: Coding and Computing, I, Pp. 7 - 112004.

[15] I. Cox and M. Miller, "Electronic watermarking: the first 50 years," in IEEE Fourth Workshop on Multimedia Signal Processing, 2001, pp. $225-230$.

[16] Z. Wang, A. Bovic, H. Sheikh, and E. Simoncelli, "Image Quality Assessment: From Error Visibility to Structural Similarity," in IEEE Transactions on Image Processing, 2004, pp. 600-612.

[17] http://www.mathworks.in/help

[18] R.G. Gonzalez and R.E. Woods, Digital Image Processing, $3^{\text {rd }}$ ed. Publishing House of Electronics Industry. 